\title{
Leucine Meal Increases Glutamine and Total Nitrogen Release from Forearm Muscle
}

\author{
ThOMAS T. AOKI, Howard Hughes Medical Institute, Joslin Research Laboratory, \\ Boston, Massachusetts 02215 \\ MuRRAy F. BRENNAN, National Cancer Institute, Bethesda, \\ Maryland 20014 \\ Gary F. FitzPatrick, Boston University School of Medicine, Division \\ of Surgery, University Hospital, Boston, Massachusetts 02118 \\ DAvid C. KNIGHT, Royal College of Medicine, Dublin, Ireland
}

\begin{abstract}
A B S TRACT To assess the consequences of elevated branched chain amino acid levels on alanine, glutamine, and ammonia metabolism in muscle, Lleucine meals $(14.7 \mathrm{~g})$ were consumed by six normal postabsorptive individuals. Bilateral forearm studies were performed, and the dominant arm was subjected to $15 \mathrm{~min}$ of light exercise, using a calibrated dynamometer, beginning $45 \mathrm{~min}$ after the ingestion of the meal. Large uptakes of leucine were seen across both forearm muscle beds within $30 \mathrm{~min}$ of the meal. After exercise, blood flow in the dominant arm increased from $3.1 \pm 0.4$ to $5.2 \pm 0.9 \mathrm{ml} / 100 \mathrm{ml}$ forearm per minute (mean \pm SEM, $P<0.005$ ). Glutamine flux out of the dominant forearm increased threefold after the ingestion of the leucine meal and increased eightfold over base line after exercise. Less marked changes (significant only at $90 \mathrm{~min}$ ) in the nonexercised, nondominant arm were also seen. Alanine flux out of the dominant forearm muscle bed increased modestly at 75 and $90 \mathrm{~min}$. No significant change in ammonia flux across either forearm muscle bed was noted. Unexpectedly, a large and significant net nitrogen loss from both forearm muscle beds was documented. Thus, following the ingestion of a leucine meal and light exercise, the primary means by which excess nitrogen is routed out of muscle is via glutamine formation and release with alanine and ammonia pathways playing relatively minor roles. More importantly, the ingestion of significant amounts of leucine by normal subjects, presumably in optimal nitrogen balance, results in a net loss of nitrogen from muscle.
\end{abstract}

Received for publication 24 November 1980 and in revised form 17 August 1981.

\section{INTRODUCTION}

When normal postabsorptive subjects consume a $200-\mathrm{g}$ broiled ground sirloin meal, systemic levels of the branched chain amino acids rise dramatically and, in turn, are removed by muscle (1-3). Because other amino acids necessary for protein synthesis are not simultaneously taken up by this tissue (muscle), it has been presumed that net protein synthesis does not take place unless intracellular amino acid pools are used in support of this activity. More recently, however, L-leucine has been infused into fasted subjects and was reported to have diminished net urinary nitrogen excretion and decreased circulating plasma amino acid levels without altering urinary 3-methylhistidine excretion. These data were interpreted as suggesting stimulation of muscle protein synthesis in these subjects (4).

The purpose of our inquiry was to address in part the above observations, using substrate flux data across normal forearm muscle bed, and to attempt to determine the fate as well as describe the metabolic impact of branched chain amino acid entry, in large quantities, on muscle. Secondly, studies of forearm muscle metabolism have traditionally been performed with this extremity relatively immobilized (5). Because mobilization may have metabolically important consequences, one forearm was lightly exercised during the study so that the influence of muscle activity on muscle metabolism could be observed. For these reasons, six normal postabsorptive subjects were asked to undergo bilateral forearm procedures in which blood samples were drawn across both forearm muscle beds before and at 30-min intervals after the ingestion of a 14.7-g L-leucine meal. 


\section{METHODS}

Six normal male subjects ranging in age between 21 and 31 yr and weighing within $\pm 6 \%$ of their ideal body weight (Metropolitan Life Tables), were carefully examined and screened with an intravenous glucose tolerance test, a chemistry profile, and triiodothyronine, reverse triiodothyronine, thyroxine, and thyrotropin determinations to ensure normality. They were then asked to report to the Clinical Research Area of the Joslin Diabetes Center (Boston, Mass.) after an overnight fast. After they had read and signed the informed consent form, the subjects were asked to rest quietly on a bed. A plastic catheter was inserted percutaneously into the nondominant(nonexercised) radial artery, and separate catheters were inserted in a retrograde direction into each antecubital vein so that blood draining forearm muscle might be selectively sampled. A pediatric blood pressure cuff was applied to each wrist and inflated to $300 \mathrm{~mm}$ of mercury $1 \mathrm{~min}$ before sampling in order to isolate the hand from the bloodsampling circuit.

Blood flow was measured simultaneously in both the exercised (dominant) and nonexercised (nondominant) arms using two complete and separate capacitance plethysmographic systems (6-8). Separate blood pressure cuffs were applied to each upper arm and briefly inflated to $40 \mathrm{~mm}$ of mercury during the blood flow determinations. This technique, first described in 1959 by Figar (6), has been in use at this laboratory for the past $5 \mathrm{yr}$ and has been found to be reliable, simple, reproducible, and accurate. The results obtained with the use of this device have been quite similar to those obtained using other plethysmographs, including both water and air displacement types, and also are in good agreement with direct invasive techniques (9) for measuring blood flow, which includes a variety of blood flow meters. Interestingly, measurements of perfusate flow in amputated legs, using instrumentation identical to those used in this investigation, were found to be in remarkably close agreement with the actually measured perfusate flow (10).

Blood samples were obtained simultaneously from all three catheters during the course of the investigation at $15-30-\mathrm{min}$ intervals and were assayed for levels of glucose (11), lactate (12), pyruvate (13), citrate (14), ammonia (15), $\beta$-hydroxybutyric acid (16), acetoacetic acid (17), glutamine (1), glutamate (1), and glycerol (18). Serum insulin (19) and plasma glucagon $(20,21)$, using $30 \mathrm{~K}$ antiserum, levels were also determined. Levels of amino acids, other than glutamine and glutamate, were determined in whole blood samples, deproteinized with $10 \%$ sulfosalicylic acid (wt/vol), and were performed on a Beckman 119 amino acid analyzer (Beckman Instruments, Inc., Fullerton, Calif.) that had been modified to permit automatic sequential injection of samples onto two discrete columns, each containing DC6A resin (bed height: $27.5 \times 0.9 \mathrm{~cm}$, Dionex Corp., Sunnyvale, Calif.). The two columns were run concurrently, and lithium buffers were used exclusively (Pico Buffer System IV, Dionex Corp.). Total run times of $\sim 4 \mathrm{~h}$ were routinely obtained with excellent resolution of all of the amino acids, as well as glutathione. The use of this resin and the lithium buffer system obviated the necessity of treating the whole blood fitrates with sodium sulfite as reported by this laboratory (1).

A single lamp photometer was constructed that provided light to the two sets of 570- and 440-nm cuvette-photocell units that were arranged around the lamp in the form of a square (MER Chromatographic, Mt. View, Calif.). The recorder of the 119 was rewired to monitor the $570-\mathrm{nm}$ cuvette of each system. However, the photocell outputs of the 570- and the 440-nm cuvettes of each system were directly routed to the separate control modules of a System AA computer (Beckman
Instruments, Inc., Spinco Div., Palo Alto, Calif.). Although it was not possible to record the $440-\mathrm{nm}$ outputs for each system due to the limitations of the recorder, both 570- and 440-nm photocell outputs were delivered to a separate control module. It was necessary to terminate the 440-nm output from the control module with a 1,000 -ohm resistor in order to maximize 440-nm signals to the control module.

The overall protocol was as follows: After the basal blood samples had been collected, the subjects swallowed a slurry containing $14.7 \mathrm{~g}$ of L-leucine (Calbiochem-Boehringer Corp., American Hoechst, San Diego, Calif.) and water over a 1-min period. Subsequent blood samples were drawn at $30,45,60$, $75,90,120$, and 150 min after the ingestion of the meal. Exercise of the dominant forearm of each subject was initiated 45 min after the ingestion of the leucine meal and consisted of maintaining a $10-\mathrm{kg}$ pull on a calibrated dynamometer (Asimow Engineering Co., Los Angeles, Calif.) for a 5-s period followed by a 5-s rest period, with the entire sequence being repeated over the next $15 \mathrm{~min}$.

Statistical analyses were performed using the Student's $t$ test (22) for paired groups (0 vs. other time points).

\section{RESULTS}

Basal blood flow determinations (Fig. 1) in both the dominant and nondominant forearms do not differ significantly with those reported by this laboratory using dye dilution techniques (5). Although resting blood flow in the dominant arm was slightly higher than that of the nondominant forearm, this difference was not significant. Blood flow did not change in the nondominant (nonexercised) arm during the entire study (Fig. 1). In contrast, blood flow in the dominant forearm (exercised) increased significantly after the completion of the exercise sequence, from basal levels of $3.1 \pm 0.4$ $\mathrm{ml} / 100 \mathrm{ml}$ forearm per $\min$ to $5.2 \pm 0.9 \mathrm{ml} / 100 \mathrm{ml}$ forearm per $\min (P<0.005)$. Blood flow in the dominant arm remained elevated for the remainder of the study. It is important to note that the above blood flow data includes blood flow through superficial tissues as well as muscle and for this reason may result in a small over estimation of net flux into and out of muscle. Parenthetically, all values shown are the mean \pm SEM. In addition, the term uptake will be used to describe a positive arterial-deep venous difference, and release will be used to describe a negative arterialdeep venous difference. The term flux will be used to describe the product of the arterial-deep venous substrate difference multiplied by flow and expressed as $\mathrm{nmol} / 100 \mathrm{ml}$ forearm per min.

Shortly after the ingestion of the leucine meal, serum insulin levels (Fig. 1) increased from $10 \pm 1$ to $18 \pm 2$ $\mu \mathrm{U} / \mathrm{ml}(30 \mathrm{~min})$, declining thereafter though remaining significantly elevated at $45(P<0.025), 60(P<0.025)$, and $75(P<0.05)$ min after the ingestion of the leucine meal.

Arterial plasma glucagon levels (Fig. 1) increased slightly from basal concentrations of $28 \pm 6 \mathrm{pg} / \mathrm{ml}$ plasma to $33 \pm 9 \mathrm{pg} / \mathrm{ml}$ within $30 \mathrm{~min}$ and did not vary significantly during the remainder of the study period. 


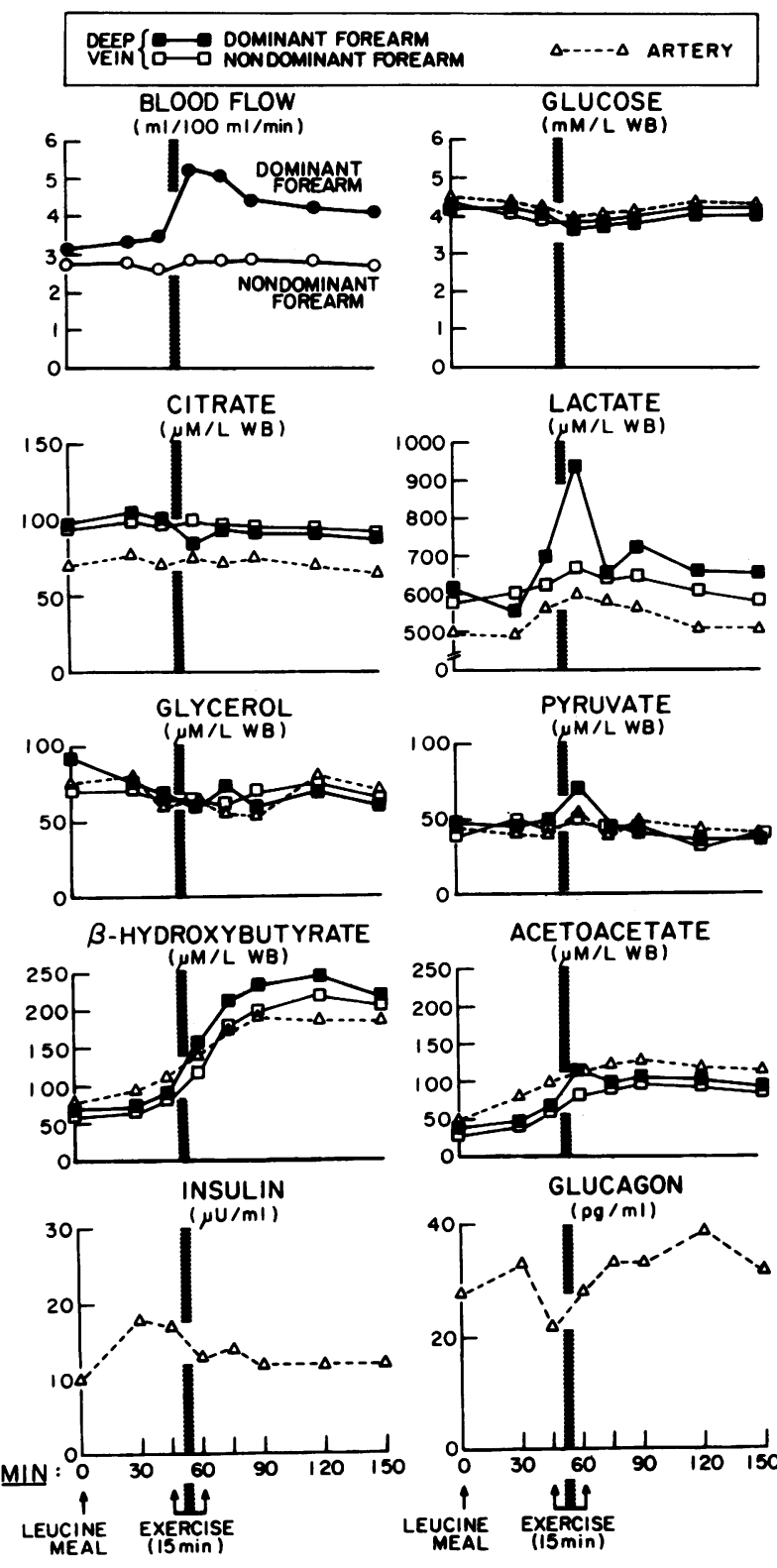

Figure 1 Blood flow, hormones, and substrates across forearm muscle beds after the ingestion of a leucine meal.

Arterial blood glucose levels (Fig. 1) decreased from $4.5 \pm 0.4$ to a nadir of $3.9 \pm 0.4 \mathrm{mM}$ at $60 \mathrm{~min}$. A significant uptake of glucose was seen across both forearms at the start of the study. Arterial-deep venous (A-DV) ${ }^{1}$ glucose differences did not change significantly across either arm at any time during the study.

Arterial blood lactate levels (Fig. 1) increased from $0.497 \pm 0.040$ to a peak concentration of $0.597 \pm 0.099$

\footnotetext{
${ }^{1}$ Abbreviation used in this paper: A-DV, arterial-deep venous.
}

$\mathrm{mM}$ at 60 min. Negative A-DV lactate differences across both forearms did not change significantly during the study.

Basal arterial blood pyruvate level (Fig. 1) was $0.045 \pm 0.005 \mathrm{mM}$. A small but significant increased release of pyruvate from the nondominant forearm muscle bed was observed at $30 \mathrm{~min}(+0.003 \pm 0.003$ vs. $-0.007 \pm 0.003 \mathrm{mM}, P<0.0025)$ and $75 \mathrm{~min}(-0.003$ $\pm 0.003 \mathrm{mM}, P<0.05)$. The pyruvate A-DV difference across dominant forearm muscle bed increased from $-0.002 \pm 0.004$ to $-0.018 \pm 0.009 \mathrm{mM}(P<0.05)$ at 60 min and converted to an uptake at $120 \mathrm{~min}(+0.007$ $\pm 0.004 \mathrm{mM}, P<0.05)$.

Arterial blood glycerol levels (Fig. 1) declined from basal values of $0.075 \pm 0.007$ to a nadir of $0.055 \pm 0.006$ $\mathrm{mM}$ at $75 \mathrm{~min}$. The A-DV differences for glycerol across the nondominant forearm muscle bed differed significantly from resting values only at $45 \mathrm{~min}(+0.005$ \pm 0.005 vs. $-0.004 \pm 0.004 \mathrm{mM}, P<0.05)$. The A-DV blood glycerol differences across the dominant forearm muscle bed differed significantly from resting values $(-0.017 \pm 0.009 \mathrm{mM})$ at $30(+0.001 \pm 0.007 \mathrm{mM}, P$ $<0.03), 60(+0.008 \pm 0.004 \mathrm{mM}, P<0.01), 120(+0.012$ $\pm 0.005 \mathrm{mM}, P<0.005)$ and $150 \mathrm{~min}(+0.011 \pm 0.007$ $\mathrm{mM}, P<0.0125)$.

Arterial blood citrate levels (Fig. 1) did not change significantly during the study. A significant release of citrate from both forearm muscle beds was observed at all time intervals with the exception of the 60-min time period across the dominant forearm muscle bed. Resting release of citrate across nondominant forearm muscle bed was $-0.026 \pm 0.004 \mathrm{mM}$ and was -0.027 $\pm 0.006 \mathrm{mM}$ across dominant forearm muscle bed. Citrate release from dominant forearm muscle bed appeared to be relatively fixed because at $60 \mathrm{~min}$, at which time blood flow had doubled, the A-DV difference of citrate across that muscle bed decreased by one half to $-0.010 \pm 0.006 \mathrm{mM}(P<0.05)$, whereas shortly thereafter, i.e. at $75 \mathrm{~min}$, citrate release was back to control values.

Reflecting the ketogenic nature of the leucine molecule, $\beta$-hydroxybutyric acid levels (Fig. 1) increased in arterial blood from $0.077 \pm 0.012$ to a peak of $0.191 \pm 0.019 \mathrm{mM}$ at $90 \mathrm{~min}$. A-DV $\beta$-hydroxybutyric acid differences increased $(P<0.05)$ over basal values $(+0.015 \pm 0.007 \mathrm{mM})$ in the nondominant arm at 30 $+0.027 \pm 0.005 \mathrm{mM})$ and $60(+0.025 \pm 0.004 \mathrm{mM}) \mathrm{min}$ and then became negative $(P<0.025)$ at $120(-0.032$ $\pm 0.014 \mathrm{mM})$ and $150(-0.009 \pm 0.013 \mathrm{mM}) \mathrm{min}$. Across the dominant forearm, increased removal of this fuel over base line $(+0.010 \pm 0.008)$ was seen at $30(+0.025$ $\pm 0.005 \mathrm{mM}, P .<0.01)$ and $45(+0.022 \pm 0.008 \mathrm{mM}$, $P<0.01) \mathrm{min}$; however at $75,90,120$ and $150 \mathrm{~min}$, removal changed to a significant $(P<0.025)$ release $(-0.038 \pm 0.010,-0.038 \pm 0.011,-0.051 \pm 0.014$, and $-0.037 \pm 0.013 \mathrm{mM}$, respectively). 
TABLE I

Arterial Levels* and Amino Acid Flux $₫$ across Both Forearm Muscle Beds of Six Normal

Volunteers before and after a Leucine Meal\$

\begin{tabular}{|c|c|c|c|c|c|c|c|c|c|c|c|c|}
\hline Time & Forearm & Taurine & $\begin{array}{l}\text { Aspartic } \\
\text { acid }\end{array}$ & Threonine & Serine & Asparagine & Glutamine & $\begin{array}{l}\text { Glu- } \\
\text { tamate }\end{array}$ & Proline & Glycine & Alanine & $\begin{array}{l}\text { Citrul- } \\
\text { line }\end{array}$ \\
\hline \multicolumn{13}{|c|}{$\min$} \\
\hline 0 & $\begin{array}{l}\text { Artery } \\
\text { Nondominant } \\
\text { Dominant }\end{array}$ & $\begin{array}{r}199 \pm 15 \\
27 \pm 30 \\
22 \pm 47\end{array}$ & $\begin{array}{c}122 \pm 8 \\
22 \pm 16 \\
22 \pm 16\end{array}$ & $\begin{array}{c}125 \pm 5 \\
-39 \pm 16 \\
-40 \pm 13\end{array}$ & $\begin{array}{r}131 \pm 6 \\
4 \pm 11 \\
-13 \pm 18\end{array}$ & $\begin{array}{c}61 \pm 4 \\
-23 \pm 11 \\
-34 \pm 20\end{array}$ & $\begin{array}{r}692 \pm 10 \\
-135 \pm 60 \\
-68 \pm 23\end{array}$ & $\begin{array}{c}179 \pm 12 \\
71 \pm 9 \\
73 \pm 14\end{array}$ & $\begin{array}{l}207 \pm 14 \\
-51 \pm 23 \\
-15 \pm 35\end{array}$ & $\begin{array}{l}285 \pm 7 \\
-37 \pm 30 \\
-30 \pm 31\end{array}$ & $\begin{array}{c}240 \pm 8 \\
-101 \pm 43 \\
-121 \pm 33\end{array}$ & $\begin{array}{c}46 \pm 4 \\
-17 \pm 9 \\
-10 \pm 14\end{array}$ \\
\hline 30 & $\begin{array}{l}\text { Artery } \\
\text { Nondominant } \\
\text { Dominant }\end{array}$ & $\begin{array}{r}193 \pm 21 \\
-39 \pm 47 \\
-20 \pm 29\end{array}$ & $\begin{array}{c}128 \pm 11 \\
-1 \pm 6 \\
8 \pm 6\end{array}$ & $\begin{array}{l}123 \pm 9 \\
-82 \pm 10^{\prime \prime} \\
-90 \pm 26^{\prime \prime}\end{array}$ & $\begin{array}{l}135 \pm 9 \\
-19 \pm 7 \\
-36 \pm 16\end{array}$ & $\begin{array}{c}61 \pm 2 \\
-36 \pm 13 \\
-39 \pm 20\end{array}$ & $\begin{array}{c}836 \pm 50 \\
-194 \pm 87 \\
-341 \pm 131\end{array}$ & $\begin{array}{r}177 \pm 10 \\
46 \pm 12 \\
61 \pm 32\end{array}$ & $\begin{array}{l}203 \pm 12 \\
-93 \pm 27 \\
-71 \pm 29\end{array}$ & $\begin{array}{l}286 \pm 11 \\
-76 \pm 14 \\
-48 \pm 24\end{array}$ & $\begin{array}{c}221 \pm 11^{*} \\
-141 \pm 23 \\
-123 \pm 23\end{array}$ & $\begin{array}{c}44 \pm 2 \\
-2 \pm 8 \\
3 \pm 13\end{array}$ \\
\hline 45 & $\begin{array}{l}\text { Artery } \\
\text { Nondominant } \\
\text { Dominant }\end{array}$ & $\begin{array}{r}203 \pm 16 \\
1 \pm 36 \\
3 \pm 25\end{array}$ & $\begin{array}{c}121 \pm 9 \\
8 \pm 7 \\
3 \pm 13\end{array}$ & $\begin{array}{c}118 \pm 5 \\
-38 \pm 11 \\
-57 \pm 20\end{array}$ & $\begin{array}{c}126 \pm 8 \\
-24 \pm 11 \\
-49 \pm 22\end{array}$ & $\begin{array}{c}68 \pm 5 \\
-7 \pm 13 \\
-11 \pm 16\end{array}$ & $\begin{array}{c}856 \pm 55^{\prime \prime} \\
-236 \pm 82 \\
-197 \pm 45\end{array}$ & $\begin{array}{c}181 \pm 12 \\
62 \pm 5 \\
81 \pm 13\end{array}$ & $\begin{array}{l}181 \pm 5 \\
-79 \pm 15 \\
-98 \pm 31\end{array}$ & $\begin{array}{l}272 \pm 9 \\
-21 \pm 21 \\
-70 \pm 27\end{array}$ & $\begin{array}{c}225 \pm 6^{\prime \prime} \\
-114 \pm 12 \\
-125 \pm 28\end{array}$ & $\begin{array}{c}42 \pm 4 \\
-8 \pm 32 \\
-17 \pm 8\end{array}$ \\
\hline 60 & $\begin{array}{l}\text { Artery } \\
\text { Nondominant } \\
\text { Dominant }\end{array}$ & $\begin{array}{c}175 \pm 21 \\
-53 \pm 29 \\
-162 \pm 116\end{array}$ & $\begin{array}{r}125 \pm 12 \\
29 \pm 17 \\
50 \pm 30\end{array}$ & $\begin{array}{c}115 \pm 5 \\
-43 \pm 13 \\
-29 \pm 26\end{array}$ & $\begin{array}{r}126 \pm 9 \\
-4 \pm 13 \\
8 \pm 18\end{array}$ & $\begin{array}{c}59 \pm 2 \\
-27 \pm 8 \\
-39 \pm 26\end{array}$ & $\begin{array}{c}866 \pm 4^{\prime \prime} \\
-211 \pm 119 \\
-217 \pm 63^{\prime \prime}\end{array}$ & $\begin{array}{c}183 \pm 12 \\
63 \pm 7 \\
64 \pm 11\end{array}$ & $\begin{array}{l}181 \pm 11 \\
-28 \pm 31 \\
-21 \pm 9\end{array}$ & $\begin{array}{r}259 \pm 13 \\
-57 \pm 11 \\
-41 \pm 32\end{array}$ & $\begin{array}{r}227 \pm 16 \\
-100 \pm 20 \\
-145 \pm 36\end{array}$ & $\begin{array}{r}34 \pm 7 \\
2 \pm 7 \\
5 \pm 4\end{array}$ \\
\hline 75 & $\begin{array}{l}\text { Artery } \\
\text { Nondominant } \\
\text { Dominant }\end{array}$ & $\begin{array}{r}197 \pm 21 \\
-3 \pm 15 \\
-27 \pm 45\end{array}$ & $\begin{array}{l}121 \pm 10 \\
-2 \pm 2 \\
-3 \pm 9\end{array}$ & $\begin{array}{c}110 \pm 6 \\
-62 \pm 25 \\
-124 \pm 31^{\prime \prime}\end{array}$ & $\begin{array}{r}123 \pm 10 \\
-17 \pm 16 \\
-50 \pm 21\end{array}$ & $\begin{array}{c}62 \pm 4 \\
-21 \pm 10 \\
-65 \pm 22\end{array}$ & $\begin{array}{c}857 \pm 51^{\prime \prime} \\
-321 \pm 108 \\
-550 \pm 75^{\prime \prime}\end{array}$ & $\begin{array}{c}182 \pm 14 \\
48 \pm 7 \\
80 \pm 15\end{array}$ & $\begin{array}{c}166 \pm 8^{\prime \prime} \\
-78 \pm 18 \\
-131 \pm 48\end{array}$ & $\begin{array}{c}262 \pm 15 \\
-50 \pm 7 \\
-119 \pm 47\end{array}$ & $\begin{array}{c}217 \pm 11^{\prime \prime} \\
-138 \pm 33 \\
-267 \pm 28\end{array}$ & $\begin{array}{c}42 \pm 5 \\
-10 \pm 4 \\
-54 \pm 24\end{array}$ \\
\hline 90 & $\begin{array}{l}\text { Artery } \\
\text { Nondominant } \\
\text { Dominant }\end{array}$ & $\begin{array}{r}213 \pm 16 \\
25 \pm 35 \\
27 \pm 32\end{array}$ & $\begin{array}{c}117 \pm 8 \\
-1 \pm 4 \\
-30 \pm 36\end{array}$ & $\begin{array}{l}104 \pm 4 " \\
-41 \pm 4 \\
-83 \pm 36\end{array}$ & $\begin{array}{r}122 \pm 5^{n} \\
2 \pm 12 \\
-22 \pm 32\end{array}$ & $\begin{array}{c}62 \pm 2 \\
3 \pm 12^{\prime \prime} \\
-5 \pm 8\end{array}$ & $\begin{array}{c}839 \pm 47^{\prime \prime} \\
-382 \pm 70^{\prime \prime} \\
-448 \pm 134^{\prime \prime}\end{array}$ & $\begin{array}{c}185 \pm 14 \\
52 \pm 8 \\
75 \pm 17\end{array}$ & $\begin{array}{c}179 \pm 6^{n} \\
-24 \pm 14 \\
-40 \pm 13\end{array}$ & $\begin{array}{l}267 \pm 9 \\
-36 \pm 11 \\
-63 \pm 42\end{array}$ & $\begin{array}{r}219 \pm 15 \\
-127 \pm 23 \\
-189 \pm 46^{\prime \prime}\end{array}$ & $\begin{array}{c}44 \pm 3 \\
11 \pm 4 \\
5 \pm 36\end{array}$ \\
\hline 120 & $\begin{array}{l}\text { Artery } \\
\text { Nondominant } \\
\text { Dominant }\end{array}$ & $\begin{array}{r}176 \pm 15 \\
-31 \pm 12 \\
-89 \pm 36\end{array}$ & $\begin{array}{r}122 \pm 12 \\
4 \pm 24 \\
6 \pm 32\end{array}$ & $\begin{array}{c}100 \pm 3^{\prime \prime} \\
-43 \pm 17 \\
-100 \pm 31^{\prime \prime}\end{array}$ & $\begin{array}{r}127 \pm 8 \\
0 \pm 19 \\
-54 \pm 31\end{array}$ & $\begin{array}{c}59 \pm 5 \\
-28 \pm 16 \\
-46 \pm 27\end{array}$ & $\begin{array}{c}853 \pm 43^{\prime \prime} \\
-348 \pm 93 \\
-21 \pm 212\end{array}$ & $\begin{array}{r}189 \pm 15 \\
49 \pm 10 \\
104 \pm 45\end{array}$ & $\begin{array}{r}166 \pm 12 \\
-54 \pm 16 \\
-114 \pm 53\end{array}$ & $\begin{array}{l}208 \pm 14 \\
-35 \pm 29 \\
-93 \pm 56\end{array}$ & $\begin{array}{r}214 \pm 17 \\
-153 \pm 32 \\
-122 \pm 8\end{array}$ & $\begin{array}{c}42 \pm 3 \\
-17 \pm 10 \\
-40 \pm 28\end{array}$ \\
\hline 150 & $\begin{array}{l}\text { Artery } \\
\text { Nondominant } \\
\text { Dominant }\end{array}$ & $\begin{array}{c}182 \pm 19 \\
-44 \pm 20^{\prime \prime} \\
-91 \pm 40 \\
\alpha-A m i n o- \\
N- \\
\text { butyrate }\end{array}$ & $\begin{array}{c}116 \pm 15 \\
-12 \pm 6 \\
-7 \pm 7\end{array}$ & $\begin{array}{c}97 \pm 7 \\
-35 \pm 12 \\
-44 \pm 21\end{array}$ & $\begin{array}{r}129 \pm 13 \\
39 \pm 19 \\
35 \pm 52 \\
\\
\text { Iso- } \\
\text { leucine }\end{array}$ & $\begin{array}{c}65 \pm 3 \\
8 \pm 14^{\prime \prime} \\
25 \pm 22\end{array}$ & $\begin{array}{c}828 \pm 49 \\
-261 \pm 61 \\
-194 \pm 104\end{array}$ & $\begin{array}{l}184 \pm 14 \\
59 \pm 7 \\
66 \pm 6 \\
\\
\text { Pheny- } \\
\text { alanine }\end{array}$ & $\begin{array}{c}168 \pm 5^{1} \\
-10 \pm 40 \\
-11 \pm 54\end{array}$ & $\begin{array}{l}270 \pm 15 \\
-22 \pm 22 \\
-28 \pm 39\end{array}$ & $\begin{array}{r}203 \pm 19 \\
-122 \pm 14 \\
-176 \pm 44\end{array}$ & $\begin{array}{l}50 \pm 2 \\
19 \pm 12^{\prime \prime} \\
19 \pm 21\end{array}$ \\
\hline 0 & $\begin{array}{l}\text { Artery } \\
\text { Nondominant } \\
\text { Dominant }\end{array}$ & $\begin{array}{r}19 \pm 2 \\
7 \pm 4 \\
2 \pm 9\end{array}$ & $\begin{array}{r}178 \pm 17 \\
1 \pm 23 \\
-7 \pm 23\end{array}$ & $\begin{array}{l}67 \pm 19 \\
41 \pm 15 \\
34 \pm 13\end{array}$ & $\begin{array}{l}51 \pm 7 \\
-4 \pm 10 \\
12 \pm 9\end{array}$ & $\begin{array}{l}103 \pm 4 \\
-26 \pm 12 \\
-16 \pm 15\end{array}$ & $\begin{array}{r}43 \pm 2 \\
-13 \pm 5 \\
-12 \pm 4\end{array}$ & $\begin{array}{r}42 \pm 3 \\
-8 \pm 9 \\
-13 \pm 7\end{array}$ & $\begin{array}{r}86 \pm 6 \\
8 \pm 9 \\
13 \pm 9\end{array}$ & $\begin{array}{l}146 \pm 6 \\
-25 \pm 21 \\
-26 \pm 24\end{array}$ & $\begin{array}{r}77 \pm 3 \\
7 \pm 10 \\
13 \pm 15\end{array}$ & $\begin{array}{c}70 \pm 3 \\
-13 \pm 13 \\
-1 \pm 14\end{array}$ \\
\hline 30 & $\begin{array}{l}\text { Artery } \\
\text { Nondominant } \\
\text { Dominant }\end{array}$ & $\begin{array}{r}15 \pm 1 \\
-9 \pm 7 \\
-10 \pm 7\end{array}$ & $\begin{array}{r}183 \pm 12 \\
-35 \pm 13 \\
-10 \pm 39\end{array}$ & $\begin{array}{l}70 \pm 17 \\
21 \pm 21 \\
32 \pm 8\end{array}$ & $\begin{array}{l}77 \pm 8^{\prime \prime} \\
27 \pm 11^{\prime \prime} \\
39 \pm 21\end{array}$ & $\begin{array}{l}1470 \pm 67^{\prime \prime} \\
1770 \pm 152^{\prime \prime} \\
1745 \pm 468^{\prime \prime}\end{array}$ & $\begin{array}{r}44 \pm 2 \\
-28 \pm 4^{\prime \prime} \\
-33 \pm 9\end{array}$ & $\begin{array}{c}43 \pm 2 \\
-28 \pm 5^{\prime \prime} \\
-26 \pm 13\end{array}$ & $\begin{array}{c}81 \pm 7 \\
-17 \pm 4^{\prime \prime} \\
-6 \pm 11\end{array}$ & $\begin{array}{l}152 \pm 7 \\
-56 \pm 8 \\
-38 \pm 24\end{array}$ & $\begin{array}{c}78 \pm 3 \\
-6 \pm 8 \\
3 \pm 13\end{array}$ & $\begin{array}{c}77 \pm 3 \\
-28 \pm 4 \\
-11 \pm 10\end{array}$ \\
\hline 45 & $\begin{array}{l}\text { Artery } \\
\text { Nondominant } \\
\text { Dominant }\end{array}$ & $\begin{array}{r}15 \pm 2 \\
0 \pm 0 \\
-6 \pm 3\end{array}$ & $\begin{array}{l}155 \pm 21^{\prime \prime} \\
-34 \pm 21 \\
-73 \pm 27\end{array}$ & $\begin{array}{r}54 \pm 21 \\
18 \pm 13 \\
9 \pm 16\end{array}$ & $\begin{array}{c}73 \pm 7 n \\
11 \pm 11 \\
8 \pm 9\end{array}$ & $\begin{array}{c}1363 \pm 137 \\
227 \pm 592 \\
527 \pm 514\end{array}$ & $\begin{array}{l}41 \pm 2 \\
-10 \pm 6 \\
-17 \pm 11\end{array}$ & $\begin{array}{l}40 \pm 3 \\
-12 \pm 5 \\
-13 \pm 13\end{array}$ & $\begin{array}{c}83 \pm 7 \\
-9 \pm 11 \\
-35 \pm 29\end{array}$ & $\begin{array}{c}153 \pm 8 \\
-52 \pm 15 \\
-67 \pm 22\end{array}$ & $\begin{array}{c}77 \pm 3 \\
-12 \pm 6^{\prime \prime} \\
-19 \pm 5^{\prime \prime}\end{array}$ & $\begin{array}{l}82 \pm 4^{\prime \prime} \\
-27 \pm 6 \\
-32 \pm 18\end{array}$ \\
\hline 60 & $\begin{array}{l}\text { Artery } \\
\text { Nondominant } \\
\text { Dominant }\end{array}$ & $\begin{array}{r}11 \pm 3 \\
1 \pm 7 \\
-1 \pm 7\end{array}$ & $\begin{array}{l}137 \pm 17 \\
-58 \pm 16 \\
-42 \pm 15\end{array}$ & $\begin{array}{l}60 \pm 16 \\
29 \pm 7 \\
47 \pm 14\end{array}$ & $\begin{array}{c}73 \pm 11 \\
-6 \pm 7 \\
-13 \pm 11\end{array}$ & $\begin{array}{c}1484 \pm 78^{\prime \prime} \\
356 \pm 360 \\
302 \pm 336\end{array}$ & $\begin{array}{r}34 \pm 2^{\prime \prime} \\
-32 \pm 5^{\prime \prime} \\
-27 \pm 9^{\prime \prime}\end{array}$ & $\begin{array}{r}33 \pm 2 n \\
-20 \pm 4 \\
-14 \pm 3\end{array}$ & $\begin{array}{r}86 \pm 8 \\
-11 \pm 6^{\prime \prime} \\
-1 \pm 13\end{array}$ & $\begin{array}{l}147 \pm 8 \\
-80 \pm 6^{\prime \prime} \\
-58 \pm 15\end{array}$ & $\begin{array}{c}71 \pm 2 \\
-11 \pm 8 \\
-12 \pm 11\end{array}$ & $\begin{array}{c}83 \pm 6 \\
-47 \pm 5 \\
-43 \pm 14\end{array}$ \\
\hline 75 & $\begin{array}{l}\text { Artery } \\
\text { Nondominant } \\
\text { Dominant }\end{array}$ & $\begin{array}{c}12 \pm 1^{n} \\
-8 \pm 3^{\prime \prime} \\
-21 \pm 16\end{array}$ & $\begin{array}{c}123 \pm 16 \\
-56 \pm 7 " \\
-117 \pm 40^{\prime \prime}\end{array}$ & $\begin{array}{c}63 \pm 13 \\
31 \pm 4 \\
7 \pm 30\end{array}$ & $\begin{array}{c}63 \pm 9 \\
4 \pm 7 \\
11 \pm 24\end{array}$ & $\begin{array}{c}1375 \pm 38 " \\
537 \pm 133^{\prime \prime} \\
380 \pm 157 "\end{array}$ & $\begin{array}{r}34 \pm 2^{\prime \prime} \\
-21 \pm 4 \\
-30 \pm 9\end{array}$ & $\begin{array}{r}29 \pm 2^{\prime \prime} \\
-20 \pm 4 \\
-31 \pm 9\end{array}$ & $\begin{array}{c}84 \pm 8 \\
-15 \pm 4^{\prime \prime} \\
-47 \pm 30\end{array}$ & $\begin{array}{c}147 \pm 10 \\
-97 \pm 14^{\prime \prime} \\
-147 \pm 44^{\prime \prime}\end{array}$ & $\begin{array}{c}78 \pm 3 \\
-27 \pm 7 \\
-8 \pm 25\end{array}$ & $\begin{array}{r}92 \pm 12 \\
-51 \pm 13^{\prime \prime} \\
-70 \pm 24^{\prime \prime}\end{array}$ \\
\hline 90 & $\begin{array}{l}\text { Artery } \\
\text { Nondominant } \\
\text { Dominant }\end{array}$ & $\begin{array}{r}14 \pm 1^{\prime \prime} \\
4 \pm 2 \\
-13 \pm 8\end{array}$ & $\begin{array}{l}112 \pm 14^{\prime \prime} \\
-35 \pm 8 \\
-80 \pm 25\end{array}$ & $\begin{array}{c}56 \pm 18 \\
22 \pm 9 \\
3 \pm 48\end{array}$ & $\begin{array}{r}44 \pm 8 \\
1 \pm 7 \\
7 \pm 7\end{array}$ & $\begin{array}{c}1396 \pm 134 \\
152 \pm 224 \\
568 \pm 430\end{array}$ & $\begin{array}{c}32 \pm 2^{\prime \prime} \\
-15 \pm 8 \\
-23 \pm 4^{\prime \prime}\end{array}$ & $\begin{array}{r}26 \pm 1^{\prime \prime} \\
-18 \pm 4 \\
-21 \pm 5\end{array}$ & $\begin{array}{c}88 \pm 6 \\
-15 \pm 11^{\prime \prime} \\
-31 \pm 20^{\prime \prime}\end{array}$ & $\begin{array}{c}149 \pm 8 \\
-73 \pm 13^{\prime \prime} \\
-116 \pm 31^{\prime \prime}\end{array}$ & $\begin{array}{c}72 \pm 3 \\
-19 \pm 6^{\prime \prime} \\
-26 \pm 16\end{array}$ & $\begin{array}{c}89 \pm 7 \\
-30 \pm 7 \\
-31 \pm 12^{\prime \prime}\end{array}$ \\
\hline 120 & $\begin{array}{l}\text { Artery } \\
\text { Nondominant } \\
\text { Dominant }\end{array}$ & $\begin{array}{r}15 \pm 2^{4} \\
5 \pm 5 \\
8 \pm 7\end{array}$ & $\begin{array}{r}94 \pm 12^{\prime \prime} \\
-20 \pm 17 \\
-41 \pm 13\end{array}$ & $\begin{array}{r}57 \pm 23 \\
-2 \pm 21 \\
-25 \pm 42\end{array}$ & $\begin{array}{l}31 \pm 4^{\prime \prime} \\
19 \pm 11 \\
28 \pm 25\end{array}$ & $\begin{array}{r}935 \pm 168 \\
-330 \pm 188 \\
-681 \pm 584\end{array}$ & $\begin{array}{r}28 \pm 4 \\
-7 \pm 7 \\
-20 \pm 9\end{array}$ & $\begin{array}{r}24 \pm 3^{\prime \prime} \\
-7 \pm 4 \\
-18 \pm 5\end{array}$ & $\begin{array}{c}82 \pm 10 \\
-27 \pm 7 " \\
-58 \pm 24^{\prime \prime}\end{array}$ & $\begin{array}{c}144 \pm 8 \\
-79 \pm 18 \\
-120 \pm 32\end{array}$ & $\begin{array}{l}77 \pm 5 \\
-7 \pm 18 \\
6 \pm 42\end{array}$ & $\begin{array}{c}86 \pm 8 \\
-22 \pm 13 \\
-57 \pm 25\end{array}$ \\
\hline 150 & $\begin{array}{l}\text { Artery } \\
\text { Nondominant } \\
\text { Dominant }\end{array}$ & $\begin{array}{c}13 \pm 2^{4} \\
6 \pm 7 \\
9 \pm 13\end{array}$ & $\begin{array}{c}80 \pm 11^{\prime \prime} \\
-2 \pm 8 \\
1 \pm 19\end{array}$ & $\begin{array}{c}49 \pm 20 \\
-5 \pm 16^{\prime \prime} \\
1 \pm 24\end{array}$ & $\begin{array}{c}16 \pm 2^{4} \\
-30 \pm 32 \\
-54 \pm 56\end{array}$ & $\begin{array}{c}754 \pm 127 \\
-29 \pm 151 \\
-246 \pm 123\end{array}$ & $\begin{array}{r}23 \pm 2^{4} \\
-10 \pm 5 \\
-15 \pm 8\end{array}$ & $\begin{array}{c}22 \pm 2^{4} \\
-3 \pm 3 \\
9 \pm 13\end{array}$ & $\begin{array}{r}84 \pm 8 \\
-8 \pm 7 \\
-10 \pm 8\end{array}$ & $\begin{array}{l}146 \pm 8 \\
-60 \pm 11 \\
-58 \pm 8\end{array}$ & $\begin{array}{l}80 \pm 6 \\
-9 \pm 9 \\
12 \pm 17\end{array}$ & $\begin{array}{c}76 \pm 5 \\
-48 \pm 11 \\
-34 \pm 11\end{array}$ \\
\hline
\end{tabular}

* Micromolar per liter whole blood.

† Nanomolar per 100 milliliter forearm per minute.

Mean \pm SEM.

" $P \leq 0.05$, paired $t$ test, 0 vs. other time periods. 
Arterial blood acetoacetic acid levels (Fig. 1) peaked at $90 \mathrm{~min}(0.050 \pm 0.009$ vs. $0.126 \pm 0.015 \mathrm{mM})$. A significant increase in acetoacetate removal by nondominant forearm muscle bed over basal values was observed at 30 and $45 \mathrm{~min}(+0.016 \pm .005$ vs. +0.037 \pm 0.006 and $+0.034 \pm 0.004 \mathrm{mM}$, respectively). A significant $(P<0.005)$ increase in acetoacetate removal by the dominant forearm muscle bed over basal values $(+0.012 \pm 0.005 \mathrm{mM})$ was observed at $30(+0.035 \pm 0.005)$ and $45(+0.031 \pm 0.006 \mathrm{mM}) \mathrm{min}$.

Arterial blood ammonia levels increased significantly from $0.099 \pm 0.026$ to $0.119 \pm 0.020 \mathrm{mM}$ at $45 \mathrm{~min}$ $(P<0.05)$. A small but significant increase in ammonia removal by dominant forearm muscle was observed at $30 \mathrm{~min}(+0.043 \pm 0.008$ vs. $+0.057 \pm 0.012 \mathrm{mM}, P$ $<0.05$ ). Of interest, the dominant and nondominant forearm A-DV ammonia differences differed significantly at $0,30,45,60,90$, and $120 \mathrm{~min}$.

Arterial levels (Table 1) of aspartic acid, taurine, threonine, serine, asparagine, citrulline, lysine, histidine, and glutamate did not change significantly while levels of proline, glycine, alanine, $\alpha$-amino- $n$-butyric acid, valine, tyrosine, and phenylalanine declined significantly $(P<0.05$, paired $t$ test). In contrast, arginine, glutamine, and, as expected, leucine levels increased. Isoleucine levels initially increased significantly (30 and $45 \mathrm{~min}$ ) but decreased thereafter. (All amino acid values shown are micromolars [whole blood], mean \pm SEM).

After the ingestion of the meal, leucine flux into muscle increased dramatically (Table I, Fig. 2). In contrast, there was a significant $(P<0.05$, paired $t$ test, 0 vs. other times) net change toward or an absolute increase in flux at one or more time points (Table I) out of this tissue for threonine, glutamine, valine, ornithine, histidine, tyrosine, phenylalanine, lysine, arginine, taurine, and less so for $\alpha$-amino- $n$ butyrate and cystine. Alanine flux (Fig. 2) did not change significantly except at 75 and $90 \mathrm{~min}$ (dominant forearm), while ammonia flux (Fig. 2) did not change significantly at any time.

Total nitrogen flux (Fig. 3) across both forearm muscle beds was calculated by multiplying the flux value for a given amino acid by the number of nitrogen groups that it contained and then algebraically summing all the resulting nitrogen fluxes (including ammonia but not urea) so determined for a given time point for a given subject. (Values shown are $\mathrm{nmol} / 100 \mathrm{ml}$ forearm per min). Total nitrogen flux across the nondominant forearm muscle bed at rest was $-290 \pm 381 \mathrm{nmol}$. Reflecting the large uptake of leucine across forearm muscle bed at $30 \mathrm{~min}$, total nitrogen flux into muscle at that time was $+544 \pm 246 \mathrm{nmol}$. Nitrogen flux from the nondominant forearm muscle bed increased to $-682 \pm 703 \mathrm{nmol}$ at $45 \mathrm{~min},-646 \pm 425$ $\mathrm{nmol}$ at $60 \mathrm{~min},-1,207 \pm 313 \mathrm{nmol}$ at $75 \mathrm{~min}(P<0.05)$, $-1,104 \pm 428 \mathrm{nmol}$ at $90 \mathrm{~min},-1,628 \pm 421 \mathrm{nmol}$ at 120

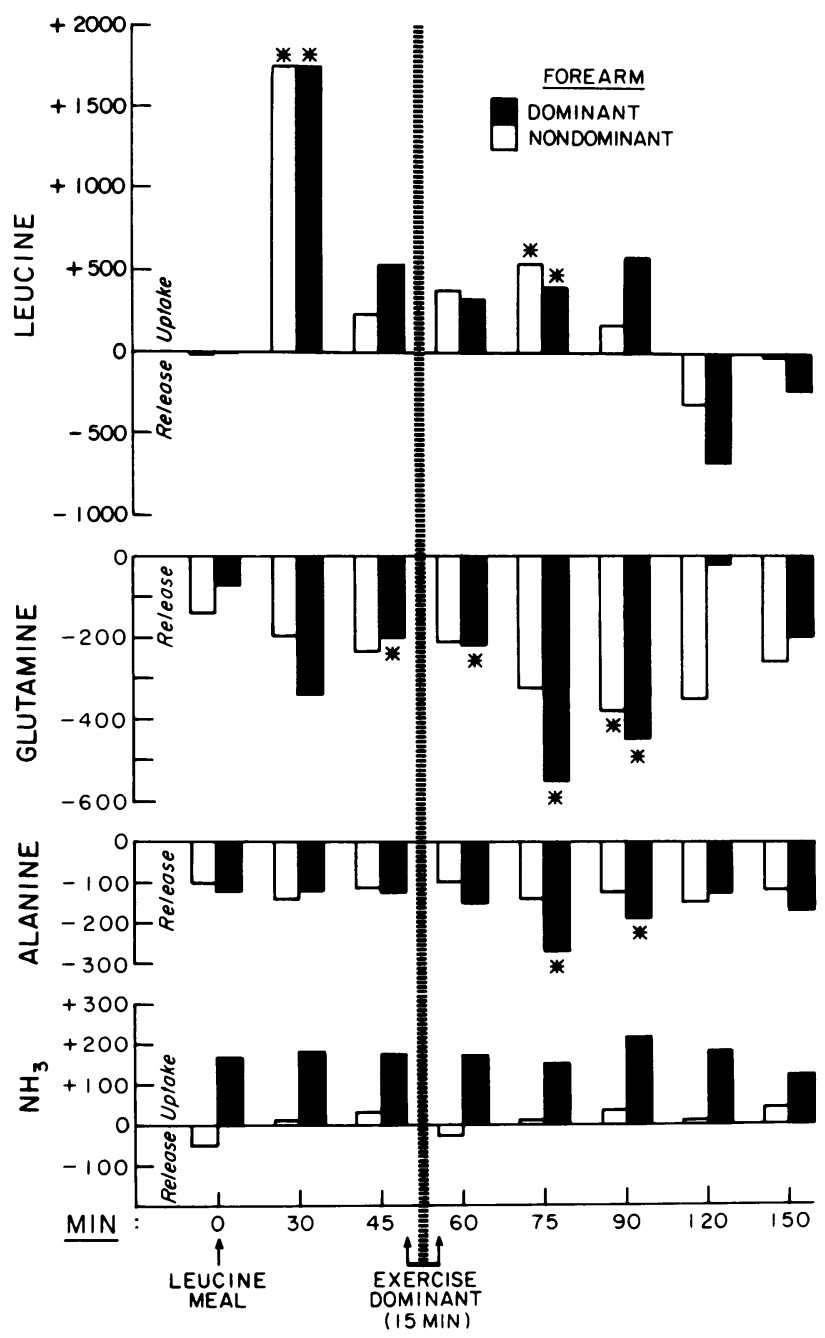

Figure 2 Leucine, glutamine, alanine, and ammonia flux ( $\mathrm{nmol} / 100 \mathrm{ml}$ forearm per min) across both dominant and nondominant forearm muscle beds following the ingestion of a leucine meal. Subsequent to leucine entry into both forearm muscle beds following ingestion of the meal, glutamine flux out of muscle increased. Alanine flux did not increase significantly except in the dominant forearm after exercise. Ammonia flux did not change across either forearm at any time. ${ }^{*}=P \leq 0.05$ or less, paired $t$ test, 0 vs. other time points.)

$\min (P<0.05)$, and $-974 \pm 261 \mathrm{nmol}$ at $150 \mathrm{~min}$. Total nitrogen flux across the dominant forearm muscle bed were as follows: $-106 \pm 377 \mathrm{nmol}$ at rest, $+646 \pm 349$ $\mathrm{nmol}$ at $30 \mathrm{~min},-535 \pm 596 \mathrm{nmol}$ at $45 \mathrm{~min},-692 \pm 274$ $\mathrm{nmol}$ at $60 \mathrm{~min},-2,531 \pm 578 \mathrm{nmol}$ at $75 \mathrm{~min},(P$ $<0.01),-1,098 \pm 389 \mathrm{nmol}$ at $90 \mathrm{~min}(P<0.05)$, $-1,819 \pm 357 \mathrm{nmol}$ at $120 \mathrm{~min}(P<0.0025)$, and -917 $\pm 291 \mathrm{nmol}$ at $150 \mathrm{~min}(P<0.05)$.

\section{DISCUSSION}

The increase in insulin (23) and the lack of change in glucagon (24) levels were as expected. Basal dominant 


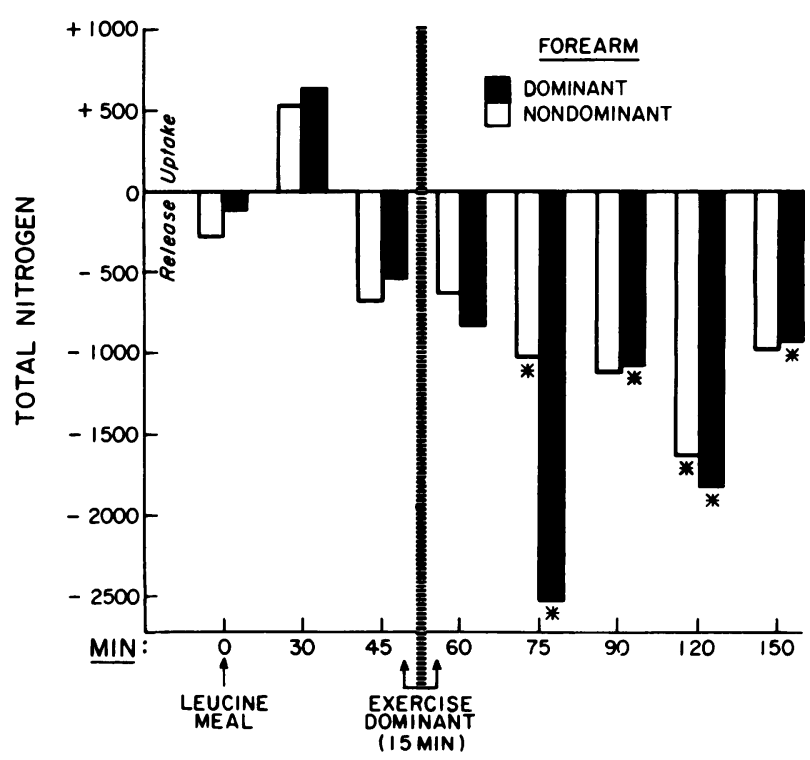

Figure 3 Total nitrogen flux $(\mathrm{nmol} / 100 \mathrm{ml}$ forearm per min) across both dominant and nondominant forearm muscle beds. Note that at 30 min there is net nitrogen entry into both forearms. However, subsequently there is significant egress of nitrogen out of both forearm muscle beds. $\left(^{*}=P\right.$ $\leq \mathbf{0 . 0 5}$ or less, paired $\boldsymbol{t}$ test, $\mathbf{0}$ vs. other time points.)

and/or nondominant muscle bed extraction of glucose, glycerol, $\beta$-hydroxybutyric and acetoacetic acids, and release of lactate and citrate is consistent with our previous experience (2) and that of others (25). Of interest, significant quantities of citrate were found to be leaving both forearm muscle beds before and after the ingestion of the leucine meal except at 60 and 90 min when A-DV differences across the dominant forearm muscle bed decreased from $-27 \pm 6$ to $-10 \pm 6$ $(P<0.05)$ and $-18 \pm 8 \mu \mathrm{M}$, respectively. The importance of citrate release by and the biochemical elucidation of its production in muscle will require further study.

The acute and dramatic elevation of circulating leucine levels followed by the initial removal and later release of this amino acid by both forearm muscle beds were of special interest. An apparent acute increase in intracellular concentration of this substrate occurred for in the face of falling arterial levels of this amino acid late in the study, a net release from muscle tissue was seen. Levels of $\alpha$-ketoisocaproic acid were not measured, but levels of this deaminated residue of leucine were almost certainly increased (26), thereby permitting other organs (liver, kidney) to participate in its oxidation.

The excess amino nitrogen removed by muscle from leucine was released not as alanine nor as ammonia but as glutamine. It was anticipated that a significant increase in alanine flux would follow massive leucine entry into muscle $(27,28)$ but increased alanine flux was seen only after the exercise sequence in the dominant arm (at 75 and $90 \mathrm{~min}, P<0.05$ ). A sig- nificant release of ammonia from muscle tissue following leucine entry was also anticipated and indeed, arterial levels increased significantly from $99 \pm 29$ to $119 \pm 20 \mu \mathrm{M}(P<0.05)$ at $45 \mathrm{~min}$. However, ammonia flux did not change across either forearm at any time.

In contrast, glutamine flux out of both forearms increased shortly after the start of the study (Fig. 2). Subsequently a marked increase in glutamine flux out of the exercised forearm was observed following completion of the exercise sequence. Thus, it would appear that the primary form by which excess amino nitrogen leaves muscle is as glutamine, which is then subsequently distributed to other tissues, including liver, kidney, and gut for differential handling. Of importance, however, is that when glutamine flux is calculated, it is not sufficient to account for the total net nitrogen flux leaving forearm muscle bed during the period of study (Fig. 3).

When total nitrogen flux into and out of forearm muscle is monitored, there is a small resting release (Fig. 3). Within $30 \mathrm{~min}$ after the ingestion of the leucine meal, there is a striking movement of nitrogen into muscle, which rapidly changes to a net flux out of this tissue. Later in the study there is a relatively large flux of nitrogen out of muscle. As noted above, this nitrogen deficit cannot be accounted for by glutamine alone. Inspection of Table I reveals that increased flux of other amino acids out of muscle can account for the additional loss of nitrogen over and above that of glutamine. Thus, the ingestion of the leucine meal resulted in the net loss of a number of amino acids from forearm muscle bed either by mobilization of intracellular amino acid pools or by an acute increase in muscle proteolysis. This finding would appear to be at odds with the in vitro work of other investigators (29), which suggest that leucine stimulates net protein synthesis in rat diaphragms. However, this difference may be due to the tissue studied as well as the very different experimental formats used. It also suggests that the expected therapeutic benefit to be derived by the infusion of relatively large quantities of the branched chain amino acids into postoperative patients may not necessarily be forthcoming (30).

Finally, this inquiry underscores the importance of a noninvasive, reproducible, and sensitive method of measuring forearm blood flow, namely capacitance plethysmography. Direct verification of the accuracy of the methodology and assumptions used are now available (10). Forearm studies with flows can now be done with relatively little effort while providing great insight into the magnitude of substrate flux changes engendered by both experimental and clinical manipulations. Also, dye techniques make some of the microfluorimetric analyses more difficult due to interference.

In summary, the ingestion of $14.7 \mathrm{~g}$ of L-leucine by six normal postabsorptive individuals resulted in the demonstration that the excess nitrogen represented by 
the test meal did not cause major changes in the uptake or release of ammonia and alanine by forearm muscle bed. Indeed, a major portion of the ingested amino nitrogen load was released from both exercised and nonexercised forearm muscle in the form of glutamine. However, the nitrogen released in the form of this amino acid could not account for the total nitrogen loss documented by complete amino acid analyses across both forearm muscle beds. This observation suggests that the leucine meal may have initiated muscle proteolysis despite a significant increase in circulating insulin levels. Further investigation into the metabolic effects of branched chain amino acid administration on muscle metabolism should be performed.

\section{ACKNOWLEDGMENTS}

We wish to acknowledge the excellent technical support provided to this project by Mmes. Adacie Allen, Patricia Hatch, Velta Ramolins, and Dzidra Rumba, and Mr. Michael Arcangeli and thank Dr. George F. Cahill, Jr. for his discussions and review of the manuscript.

This paper was supported in part by National Institutes of Health grant AM-15191, BRSG S07 RR-05673, and the Howard Hughes Medical Institute.

\section{REFERENCES}

1. Aoki, T. T., W. A. Müller, M. F. Brennan, and G. F. Cahill, Jr. 1973. Blood cell and plasma amino acid levels across forearm muscle and plasma amino acid levels across forearm muscle during a protein meal. Diabetes. 22: 768-775.

2. Aoki, T. T., J. F. Brennan, W. A. Müller, J. S. Soeldner, J. S. Alpert, S. B. Saltz, R. L. Kaufmann, M. H. Tan, and G. F. Cahill, Jr. 1976. Amino acid levels across normal forearm muscle and splanchnic bed after a protein meal. Am. J. Clin. Nutr. 29: 340-350.

3. Wahren, J., P. Felig, and J. Hagenfeldt. 1976. Effect of protein ingestion on splanchnic and leg metabolism in normal man and in patients with diabetes mellitus.J. Clin. Invest. 57: 987-999.

4. Sherwin, R. S. 1978. Effect of starvation on the turnover and metabolic response to leucine. J. Clin. Invest. 61: 1471-1481.

5. Pozefsky, T., P. Felig, J. D. Tobin, J. S. Soeldner, and G. F. Cahill, Jr. 1969. Amino acid balance across tissues of the forearm in postabsorptive man. Effects of insulin at two dose levels. J. Clin. Invest. 48: 2273-2282.

6. Figar, S. 1959. Electrocapacitance plethysmography. Physiol. Bohemoslov. 8: 275-282.

7. Wood, J. R., and C. A. Hyman. 1970. A direct reading capacitance plethysmograph. Med. Biol. Eng. 8: 59-70.

8. Wenger, C. B., M. F. Roberts, J. A. J. Stolwijk, and E. R. Nadel. 1975. Forearm blood flow during body temperature transients produced by leg exercise.J. Appl. Physiol. 38: 58-63.

9. Longhurst, J., R. J. Capone, D. T. Mason, and R. Zelis. 1974. Comparisons of blood flow measured by plethysmograph and flowmeter during steady state forearm exercise. Circulation. 49: 535-540.

10. Norton, J. A., M. E. Burt, and M. F. Brennan. 1981. Validation of blood flow rates measured by the capacitance plethysmograph. Am. J. Physiol. In press.

11. Hoffman, W. S. 1937. A rapid photometric method for the determination of glucose in blood and urine. J. Biol. Chem. 120: 51-55.

12. Passonneau, J. V. 1974. Lactate:fluorimetric method. In Methods of Enzymatic Analysis. H. U. Bergmeyer, editor. Verlag chemis weinheim. Academic Press, Inc., New York and London. 1468-1472.

13. Passonneau, J. V., and O. H. Lowry. 1974. Pyruvate. In Methods of Enzymatic Analysis. H. U. Bergmeyer, editor. Verlag chemie Weinheim. Academic Press, Inc., New York and London. 1452-1456.

14. Passonneau, J. V., and J. G. Brown. 1974. Citrate: fluorimetric determination. In Methods of Enzymatic Analysis. H. U. Bergmeyer, editor. Verlag chemie Weinheim. Academic Press, Inc., New York and London. 1565-1569.

15. Kun, E., and E. B. Kearney. 1974. Ammonia. In Methods of Enzymatic Analysis. H. U. Bergmeyer, editor. Verlag chemie Weinheim. Academic Press, Inc., New York and London. 1802-1806.

16. Williams, D. H., and J. Mellanby. 1974. D-(-)-3-Hydroxybutyrate. In Methods of Enzymatic Analysis. H. U. Bergmeyer, editor. Verlag chemie Weinheim. Academic Press, Inc., New York and London. 1836-1839.

17. Mellanby, J., and D. H. Williamson. 1974. Acetoacetate. In Methods of Enzymatic Analysis. H. U. Bergmeyer, editor. Verlag chemie Weinheim. Academic Press, Inc., New York and London. 1840-1843.

18. Wieland, O. 1974. Glycerol. In Methods of Enzymatic Analysis. H. U. Bergmeyer, editor. Verlag chemie Weinheim. Academic Press, Inc., New York and London. 1404-1409.

19. Soeldner, J. S., and D. Sloane. 1965. Critical variables in the radio-immunoassay of serum insulin using the double antibody technique. Diabetes. 14: 771-779.

20. Aguilar-Parada, E., A. M. Eisentraut, and R. H. Unger. 1969. Effects of starvation on plasma pancreatic glucagon in normal man. Diabetes. 18: 717-723.

21. Weir, G. C., R. C. Turner, and D. B. Martin. 1973. Glucagon radioimmunoassay using antiserum 30K: interference by plasma. Horm. Metab. Res. 5: 241-244.

22. Snedecor, G. W. 1956. Statistical Methods Applied to Experiments in Agriculture Biology. Iowa State College Press, Ames, Iowa, 5th edition. 45-53.

23. Floyd, J. C., Jr., S. S. Fajans, R. F. Knopf, and J. W. Conn. 1963. Evidence that insulin release is the meachanism for experimentally induced leucine hypoglycemia in man. J. Clin. Invest. 42: 1714-1719.

24. Rocha, D. M., G. R. Faloona, and R. H. Unger. 1972. Glucagon-stimulating activity of twenty amino acids in dogs. J. Clin. Invest. 51: 2346-2351.

25. Owen, O. E., and G. A. Reichard, Jr. 1971. Human forearm metabolism during progressive starvation. J. Clin. Invest. 50: 1536-1545.

26. Hutson, S. M., T. C. Cree, and A. E. Harper. 1978. Regulation of leucine and $\alpha$-ketoisocaproate metabolism in skeletal muscle. J. Biol. Chem. 253: 8126-8133.

27. Felig, P., T. Pozefsky, E. Marliss, and G. F. Cahill, Jr. 1970. Alanine: key role in gluconeogenesis. Science. (Wash. D. C.). 167: 1003-1004.

28. Odessey, R., E. A. Chairallah, and A. L. Goldbert. 1974. Origin and possible significance of alanine production by skeletal muscle. J. Biol. Chem. 249: 7623-7629.

29. Buse, M. G., and S. S. Reid. 1975. Leucine: a possible regulator of protein turnover in muscle. J. Clin. Invest. 56: $1250-1261$.

30. Freund, H., H. C. Hoover, Jr., S. Atamian, and J. E. Fisher. 1979. Infusion of the branched chain amino acids in postoperative patients. Anticatabolic properties. Ann. Surg. 190: 18-23. 